\title{
Automated Interpretation of Regional Left Ventricular Wall Motion from Cardiac Magnetic Resonance Images
}

\author{
Enrico G. Caiani, PhD, ${ }^{1,2}$ Eran Toledo, PhD, ${ }^{1}$ Peter MacEneaney, MD, ${ }^{1}$ Dianna Bardo, MD, ${ }^{1}$ Sergio Cerutti, MS, ${ }^{2}$ \\ Roberto M. Lang, MD, ${ }^{1}$ and Victor Mor-Avi, PhD ${ }^{1}$ \\ ${ }^{1}$ Noninvasive Cardiac Imaging Laboratory, University of Chicago, Chicago, Illinois, USA \\ ${ }^{2}$ Dipartimento di Bioingegneria, Politecnico di Milano, Milan, Italy
}

\begin{abstract}
Magnetic resonance (MR) diagnosis of regional left ventricular (LV) dysfunction relies on visual interpretation of cine images that suffers from wide inter-observer variability, especially when performed by readers not specifically trained in the assessment of LV wall motion. Quantitative analysis tools, though widely available, are rarely used because they provide large amounts of detailed information, the interpretation of which requires additional time-consuming processing. We tested the feasibility of fast automated interpretation of regional LV function using computer analysis of this wall motion information. Methods: Dynamic, ECG-gated, steadystate free precession short-axis images were obtained in 6-10 slices in 28 subjects ( 10 normal volunteers; 18 patients). Images were reviewed by an expert cardiologist who provided "gold standard" grades (normal, abnormal) for regional wall motion and, independently, by four radiologists. Same images were then analyzed using custom software. Regional fractional area changes computed in normal volunteers were used to obtain the optimal segment- and slicespecific threshold values for automated classification of regional wall motion for each patient. The levels of agreement with the "gold standard" grades were compared between the radiologists and the automated interpretation. Results: While the visual interpretation required 2-5 minute per patient, the automated interpretation required $<1 \mathrm{sec}$, after endocardial border detection was complete. The automated interpretation resulted in higher sensitivity, specificity, and accuracy $(84 \%, 77 \%, 79 \%$, respectively) than the radiologists' grades $(80 \%, 76 \%, 77 \%$, respectively) and eliminated the high interobserver variability. Conclusion: Once the endocardial boundaries are defined, computer analysis of the regional wall motion information allows accurate, fully automated, immediate, objective and experience-independent interpretation of regional LV function.
\end{abstract}

\section{INTRODUCTION}

Cardiac magnetic resonance (CMR) evaluation of global left ventricular (LV) function is routinely performed based on mea-

Keywords: Left Ventricle, Wall Motion, Automated Diagnosis. Correspondence to:

Victor Mor-Avi, PhD

University of Chicago, Medical Center

MC5084, 5841 S. Maryland Ave.

Chicago, Illinois 60637

tel: (773)-702-7780; fax: (773)-702-1034

email:vmoravi@medicine.bsd.uchicago.edu surements of LV volumes, ejection fraction and mass (1-3). These parameters are calculated from semi-automatically traced endocardial and epicardial boundaries using commercial software. In contrast, CMR diagnosis of regional LV dysfunction is mostly based on visual inspection of dynamic cine images of the left ventricle in multiple planes and the interpretation of regional wall motion (4-8). This interpretation relies on integrating spatial and temporal information, which is subjective and requires extensive training and experience (9-12). In clinical practice, the interpretation of CMR images for the evaluation of regional LV function is predominantly performed by radiologists who often do not have the necessary expertise to accurately diagnose regional wall motion abnormalities. Although the outcome of 
this subjective methodology may vary widely between observers (13), visual interpretation is commonly used in clinical practice despite the availability of tools for quantitative analysis of regional wall motion. This is because these quantitative analysis tools provide large amounts of detailed information, which cannot be immediately interpreted without additional processing that requires significant time commitment.

Accordingly, our goals were: (i) to test the feasibility of objective interpretation of regional LV function using automated quantitative analysis of segmental wall motion information provided by these conventional tools and (ii) to display the results of the automated interpretation in a familiar, easily understandable, visual format. This study was designed to test the performance of our technique for automated detection of regional wall motion abnormalities by comparing its results with the conventional visual interpretation of LV wall motion by an expert reader. To put these results in perspective, we also studied the accuracy and the inter-observer variability of conventional visual interpretation of regional LV function by less experienced readers on the same set of images.

\section{METHODS}

\section{Population}

The study population consisted of 28 subjects (16 male, 12 female; mean age: $50 \pm 20$ ). Ten subjects were healthy volunteers with no known history of cardiac disease. The remaining 18 subjects were patients referred for CMR imaging for the evaluation of LV function. Exclusion criteria were: dyspnea precluding a 10-15 sec breath-hold, cardiac arrhythmias, left bundle branch block, prior sternotomy, implanted pacemaker or defibrillator, claustrophobia and other known contraindications to MR imaging. Written informed consent was obtained from all study subjects.

\section{Imaging}

Images were obtained with a 1.5 Tesla scanner (General Electric, Milwaukee, Wl) with a phased-array cardiac coil. ECGgated localizing spin-echo sequences were used to identify the long-axis of the heart and thus allow imaging in the anatomically correct short-axis planes. Steady-state free precession (SSFP) dynamic gradient-echo cine-loops were obtained during 10-15 sec breath-holds with a temporal resolution of 20 frames per cardiac cycle. In all subjects, 6 to 10 short-axis cine-loops were obtained from the atrioventricular ring to the apex $(9 \mathrm{~mm}$ slice thickness, no gaps).

\section{Slice selection}

Initially, in each of the 28 study subjects, LV slices were selected for analysis beginning with the highest basal slice where the LV outflow tract was not visible and ending with the lowest apical slice where the LV cavity was visible. Because of the individual differences in the LV long-axis dimension, the number of slices selected for analysis varied between subjects.

\section{Visual interpretation of wall motion}

Dynamic images obtained in the 18 patients were reviewed by an expert cardiologist (20 years of experience in echocardiography, 3000 exams per year), blinded to the patient's identity and diagnosis. The review was based on a standard segmentation scheme used in echocardiography for grading regional LV wall motion in the short-axis view (14). In each slice, the LV myocardium was visually divided into six segments: anterior, anteroseptal, septal, inferior, posterior and lateral. Wall motion in each segment was classified as either normal or abnormal. This visual interpretation was used as the "gold standard" for segment-by-segment comparisons. Thereafter, the same dynamic images were independently reviewed and regional wall motion was graded as normal or abnormal by four radiologists with different levels of experience in the interpretation of cardiac images, ranging from level 1 to level 2 (15). During the reading, the radiologists were blinded to both the expert's and the other observers' interpretations.

\section{Computer analysis of CMR images}

Images obtained in every study subject (10 normal volunteers and 18 patients) were analyzed as follows. Since the commercial analysis software does not allow exporting the detected endocardial contours for further processing, conventional thresholdbased image segmentation technique previously described in detail (16), which was implemented in Matlab (The Mathworks Inc., Natick, MA) was used to detect LV endocardial borders in each slice throughout the cardiac cycle (Fig. 1A-C). Algorithm parameters were initially adjusted for the first frame in each image sequence by visually verifying the position of the resulting contour, which was superimposed on the original image (Fig. 1D). Then, the optimal parameters were applied to the subsequent images and adjusted when necessary to optimize border position for that particular frame.

In each slice, LV cavity area was then calculated frame-byframe directly from pixel counts inside the detected endocardial border. The LV volume was then calculated frame-by-frame as the sum of LV areas in all slices times the slice thickness. The systolic (ES) and end-diastolic (ED) frames were automatically identified as those with the smallest and the global largest LV volumes, correspondingly (Fig. 2, left), and used to define the standard segmentation scheme for the LV short-axis view in each slice (14). The ED centroid of the LV cavity was calculated automatically and used as the origin of segmentation. An additional point was then manually placed at the junction between the right ventricular free wall and the interventricular septum on the ED frame. Starting from that point, the LV cavity was divided into six $60^{\circ}$ wedge-shaped segments (Fig. 2, top middle), corresponding to those used for visual assessment and grading of wall motion. For each segment, regional fractional area (RFA) in \% of regional end-diastolic area (REDA) was calculated automatically (Fig. 2, right) throughout the cardiac cycle using a fixed-coordinate reference system. 


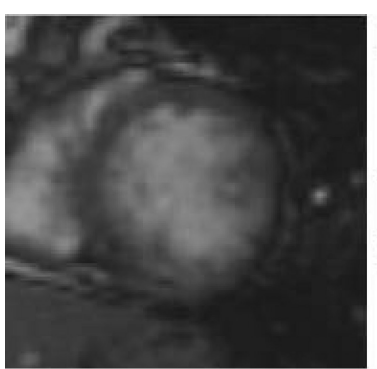

A

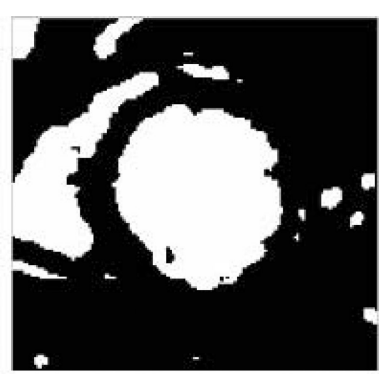

$\mathrm{B}$

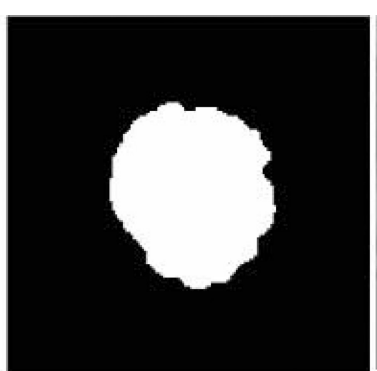

$\mathrm{C}$

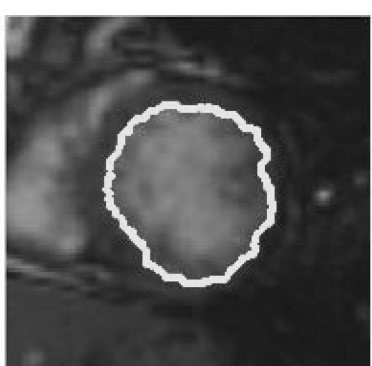

$\mathrm{D}$

Figure 1. Principles of the semi-automated LV endocardial border detection applied frame-by-frame. The original CMR cine loop was cropped to contain the LV cavity (Fig. 1A); then, a binary image was created by means of conventional threshold-based image segmentation techniques (Fig. 1B), and further processed with standard morphological operators to extract the LV cavity (Fig. 1C), while including the papillary muscles in the LV cavity as much as possible by fine-tuning algorithm parameters. Finally, the position of the resulting contour was superimposed on the original image for visual verification (Fig. 1D).

\section{Automated interpretation of wall motion}

RFAC data obtained in the 10 normal volunteers were initially used to define regional threshold values, which were subsequently used for automated detection of wall motion abnormalities in the remaining 18 patients. To allow averaging of RFAC in subjects with different number of slices covering the left ventricle from base to apex, values from different slices in each subject were resampled using cubic spline interpolation to obtain 100 values from base to apex for each LV wall (anterior, anteroseptal, septal, inferior, posterior and lateral). These values were then averaged for all normal volunteers on a point-by-point basis to obtain mean RFAC and the corresponding SD, every $1 \%$ of the LV length from base to apex. Mean minus 1SD data were plotted to obtain a normal reference curve for each of the six LV walls.

From these normal reference curves, RFAC threshold for each segment in each slice was then individually computed for each patient according to the number of slices. Each normal reference curve was divided into a number of intervals equal to the number of slices covering the left ventricle from base to apex in each patient. For each LV wall, RFAC values within each interval were averaged to represent the threshold for the corresponding slice. These thresholds were then used to automatically classify regional wall motion as normal (RFAC $\geq$ threshold) or abnormal RFAC $<$ threshold).

\section{Binary "bull's eye" summary}

To allow easy visualization of the results of the automated interpretation of regional LV wall motion in the entire ventricle in a single picture, the classification data were displayed in a binary "bull's eye" format, where darker areas represent abnormal segments. In this display, the inner circle represents the apex, while the outer rings represent consecutive slices from apex to base. In addition, to allow easy visual comparisons between the results of the automated interpretation and the "gold standard" expert interpretation, the latter results were also displayed in the same "bull's eye" format.

\section{Statistical analysis}

The individual readings of the four radiologists were compared to the gold standard by counting the segments where concordant (true positive and negative) as well as discordant (false positive and negative) readings were made. Segment counts were used to calculate the sensitivity, specificity, and overall accuracy of each reader, which were then averaged. In addition, we calculated the interobserver variability of the radiologists' interpretation which was defined in two different ways: (i) as the percentage of segments in which the interpretation of at least one of the readers was different from that of the other three (more strict criterion) and (ii) as the percentage of segments in which the interpretation of two readers was different from that of the other two (less strict criterion).

Similarly, the automated segment classification of regional LV wall motion was compared to the gold standard by counting the segments where concordant as well as discordant readings were made. Segment counts were used to calculate the sensitivity, specificity, and overall accuracy of the automated interpretation of regional wall motion.

The sensitivity, specificity, and accuracy obtained with the automated method were compared with those obtained by the four radiologists by using the Z-test with Yates correction and considered significant for $p<0.05$. For these analyses, we used sensitivity, specificity, and accuracy as sample proportions and the relevant number of segments as sample sizes.

In addition, to test the performance of our technique in patients with regional wall motion abnormalities, the results of both visual and automated interpretation were evaluated separately in a subset of patients who had wall motion abnormalities according to the gold standard expert interpretation.

\section{RESULTS}

While the visual interpretation by radiologists required 2 to 5 minutes per patient, the calculation of RFAC was fully automated and required $<1 \mathrm{sec}$ per patient ( $2 \mathrm{GHz}$ Pentium- 4 

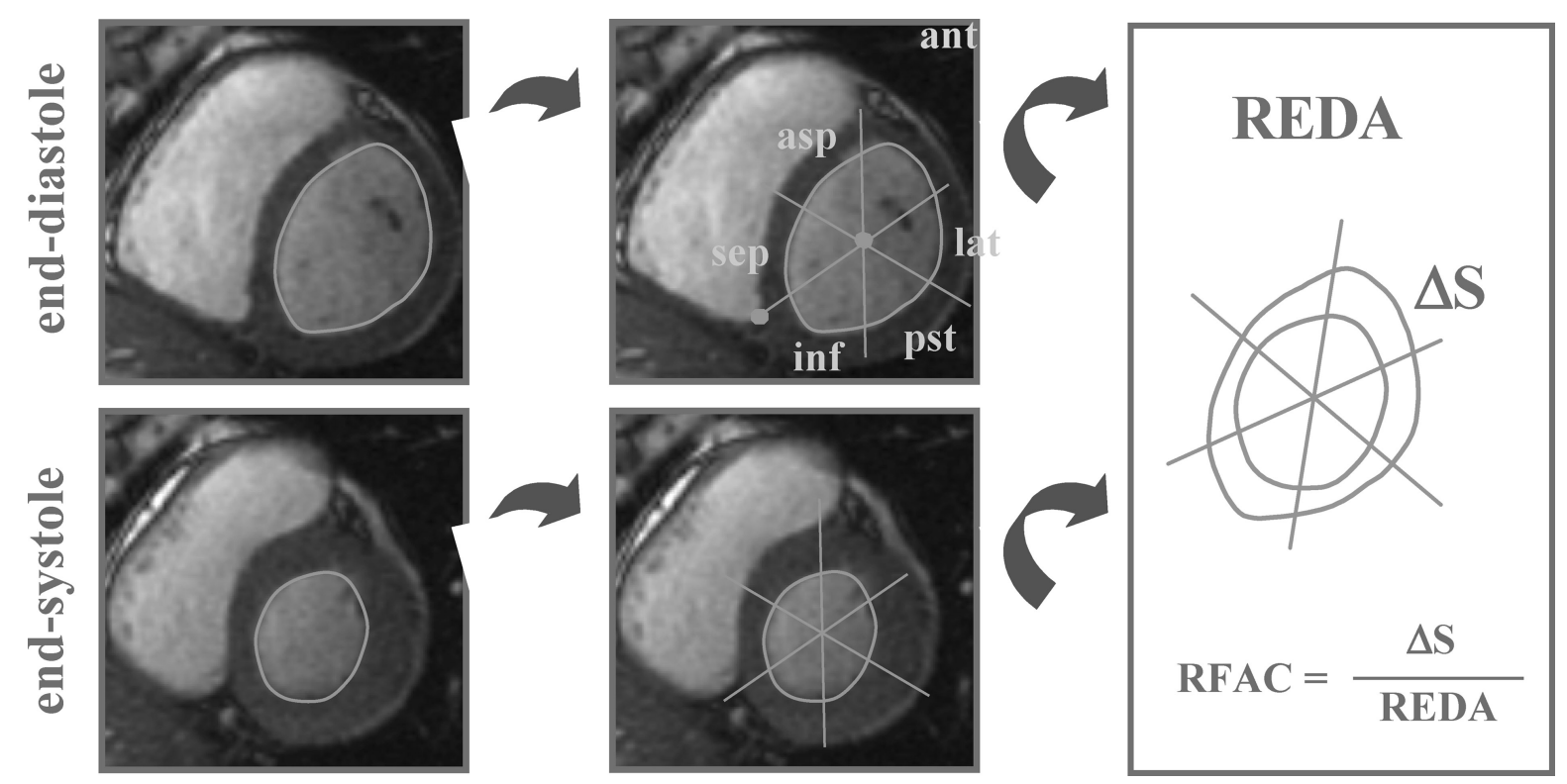

Figure 2. Schematic representation of the computation of regional fractional area change (RFAC) in a short-axis slice of the left ventricle. Following segmentation (middle column; see text for detail), end-diastolic (ED, top, left) and end-systolic (ES, bottom, left) regional LV cavity areas were automatically measured and used to calculate RFAC, which was defined as the area $\triangle S$ between the ED and ES position of the endocardial border, in \% of the regional end-diastolic area (REDA).

personal computer Dell Inc., Round Rock, TX), once the endocardial borders were detected and the anatomic landmarks required for segmentation were set, which took $20 \mathrm{sec}$ to $5 \mathrm{~min}$ per slice depending on the quality of endocardial visualization and the presence of the papillary muscles in that particular slice. Figure 3 shows an example of regional fractional area (RFA) time curves obtained in six segments of one basal LV slice in a normal volunteer. The general shape of these curves reflects on a regional basis the expected changes in $\mathrm{LV}$ volume throughout the cardiac cycle, including systolic contraction and the subsequent diastolic filling phases (rapid filling, diastasis and atrial contraction). Also, as expected, in this basal slice, in the segments adjacent to the aortic outflow tract (septal and anteroseptal),

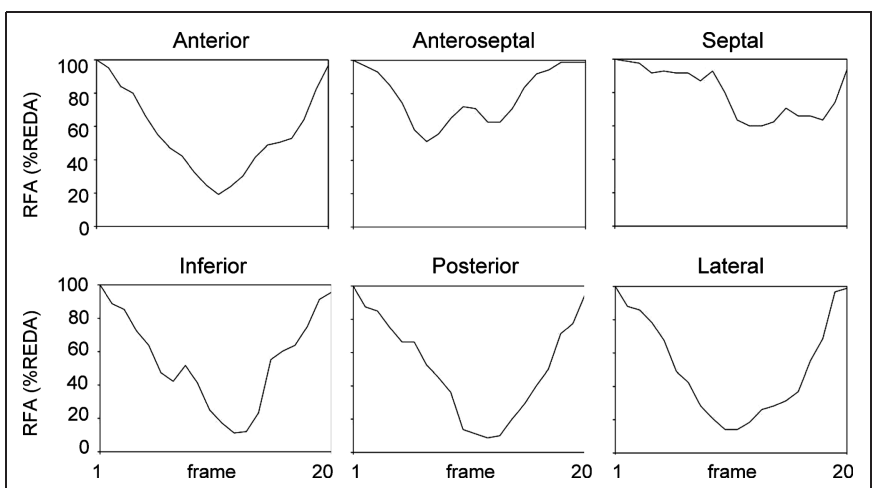

Figure 3. Example of regional fractional area (RFA) time curves, in percent of regional end-diastolic area (REDA) throughout the cardiac cycle, measured in 6 segments of a basal LV slice in a normal volunteer. the peak-to-peak amplitude of the RFA curves, ie, the regional fractional area change (RFAC), was relatively low compared to the other segments, reflecting the heterogeneity in regional LV contraction patterns.

Figure 4 shows the mean RFAC values obtained in the 10 normal volunteers at different levels of the left ventricle from base to apex, with the corresponding normal reference curves (ie, mean-1SD) below. These normal reference curves were then used to calculate the regional thresholds for automated detection of regional wall motion abnormalities in each individual patient. These curves showed an increase in RFAC from base to apex

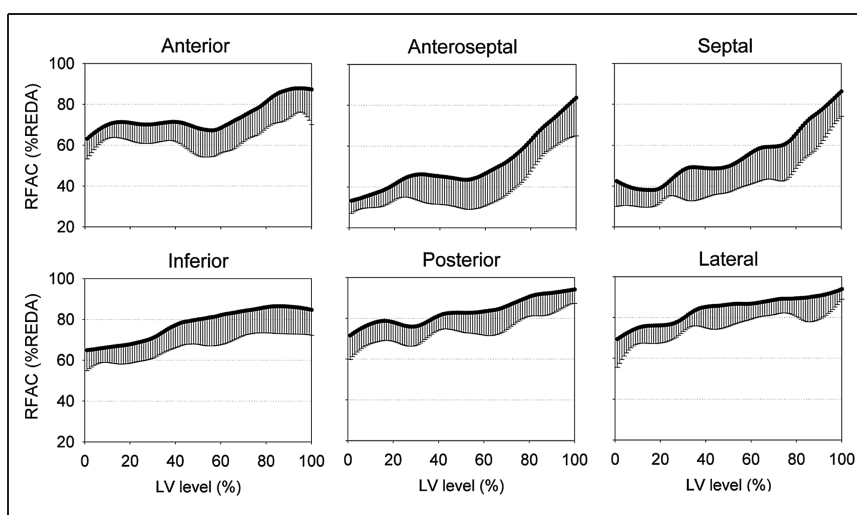

Figure 4. Mean RFAC curves (solid black lines with SD bars facing down) obtained in the 10 normal volunteers at different levels of the left ventricle from base to apex (every $1 \%$ of the LV length) in six LV walls. The corresponding normal reference curves are defined by the lower end of the SD bars. 


\section{Color-encoded RFAC}

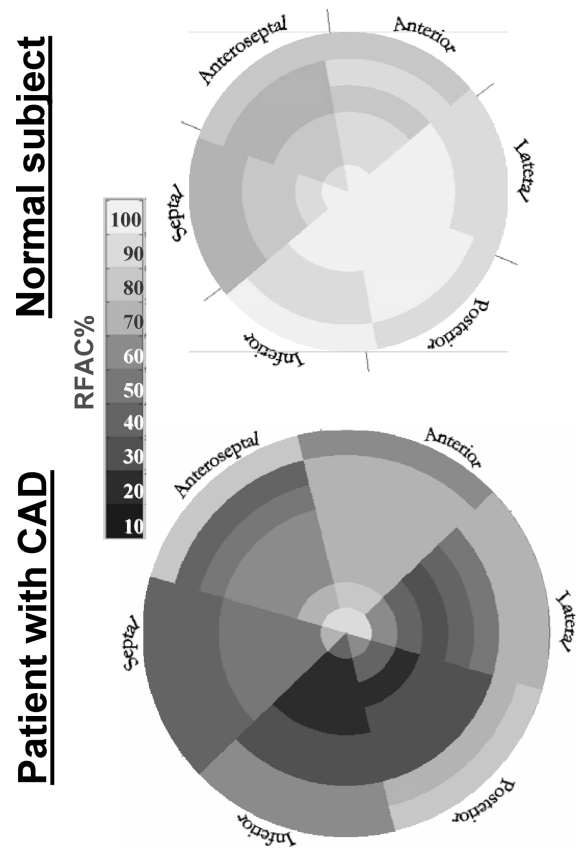

Automated classification
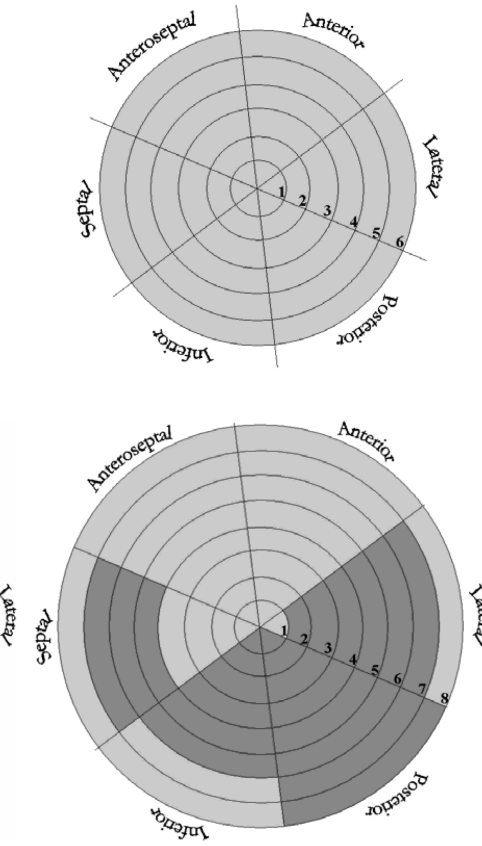

Expert interpretation

Figure 5. Example of the "bull's eye" representation of the color-encoded regional fractional area change (RFAC, left), the corresponding automated classification (middle) and the "gold standard" expert reader's grades (right), obtained in a normal subject (top) and a patient with abnormal wall motion secondary to CAD (bottom). In this display, the inner circle represents the apex (slice 1), while the outer rings represent consecutive slices ( 2 and up) from LV apex to base, and the darker areas represent segments classified as abnormal.

in all LV walls. While this increase was moderate (order of magnitude of $20 \%$ ) in the anterior, inferior, posterior and lateral walls, it was more pronounced (50 to $60 \%$ ) in the anteroseptal and septal walls, consistent with the reduced mobility of the basal LV segments adjacent to the aortic outflow tract.

According to the gold standard interpretation, of the $18 \mathrm{pa}-$ tients studied, 6 had normal LV wall motion, 10 had regional wall motion abnormalities, and 2 had global hypokinesis secondary to dilated cardiomyopathy. Of 768 segments examined (128 slices, 6 segments per slice), 541 (70\%) were classified as normal and 227 (30\%) as abnormal.

Figure 5 shows an example of data obtained in a patient with normal wall motion (top) and a patient with abnormal wall motion secondary to coronary artery disease (CAD) (bottom). In the patient with normal wall motion according to the "gold standard" interpretation (top right), the "bull's eye" display showed uniformly normal RFAC (top left) in the entire ventricle which was automatically classified as normal in all segments (top middle). In contrast, in the patient with abnormal wall motion detected by the expert (bottom right), the "bull's eye" showed darker areas reflecting reduced RFAC (bottom left), roughly in the corresponding segments, which were automatically classified as abnormal (bottom, middle).

In the entire group of 18 patients, the automated technique showed normal RFAC in 455/768 segments (59\%) and abnormally reduced RFAC in the remaining 313/768 segments
(41\%). This interpretation disagreed with the "gold standard" in $160 / 768$ segments $(21 \%)$, resulting in overall accuracy of $79 \%$. Of these 160 discordant segments, 123 were false positive, and 37 were false negative, resulting in a sensitivity of $84 \%$ and specificity of $77 \%$.

Table 1 presents the sensitivity, specificity and accuracy data calculated for the radiologists' readings and the

\begin{tabular}{|c|c|c|c|}
\hline $\begin{array}{l}\text { Radiologists' } \\
\text { interpretation }\end{array}$ & Sensitivity & Specificity & Accuracy \\
\hline Observer 1 & $72 \%(68 \%)$ & $88 \%(82 \%)$ & $83 \%(78 \%)$ \\
\hline Observer 2 & $96 \%(94 \%)$ & $48 \%(42 \%)$ & $62 \%(58 \%)$ \\
\hline Observer 3 & $89 \%(83 \%)$ & $78 \%(71 \%)$ & $81 \%(75 \%)$ \\
\hline Observer 4 & $64 \%(50 \%)$ & $89 \%(85 \%)$ & $82 \%(74 \%)$ \\
\hline Mean & $80 \%(74 \%)$ & $76 \%(70 \%)$ & $77 \%(71 \%)$ \\
\hline $\begin{array}{l}\text { Automated } \\
\text { interpretation }\end{array}$ & $84 \% *(79 \% *)$ & $77 \%(74 \% *)$ & $79 \% *(76 \% *)$ \\
\hline \multicolumn{4}{|c|}{$\begin{array}{l}\text { Sensitivity, specificity, and accuracy of the interpretation of regional LV } \\
\text { wall motion by four radiologists and by the automated classification } \\
\text { procedure (see text for details), calculated against the "gold standard" } \\
\text { expert cardiologist's interpretation ("p }<0.05 \text { radiologists' interpretation } \\
\text { vs. the automated technique). Results are reported for the entire group } \\
\text { of } 18 \text { patients and, in parentheses, for a subset of } 10 \text { patients with } \\
\text { regional wall motion abnormalities. }\end{array}$} \\
\hline
\end{tabular}


automated method, both compared to the "gold standard" interpretation. The four radiologists' interpretation resulted in a mean sensitivity of $80 \%$, specificity of $76 \%$ and accuracy of $77 \%$. These levels of agreement were lower than those of the automated interpretation (Table 1), with significance reached for sensitivity and accuracy. In the subset of 10 patients with LV regional wall motion abnormalities (Table 1, values in parentheses), the sensitivity, specificity and accuracy of the radiologists' interpretation were even lower $(74 \%, 70 \%$, and $71 \%$, respectively), which were all significantly lower than those of the automated interpretation.

In the entire group of 18 patients, the radiologists' grades were discordant in 397/768 segments $(52 \%)$ based on the more strict criterion (at least one discordant grade), and in 111/768 segments $(15 \%)$ based on the less strict criterion, reflecting the high inter-observer variability of the less experienced readers. In the subset of 10 patients with LV regional wall motion abnormalities, the interobserver variability was even higher: $58 \%$ and $20 \%$ according to the two criteria, respectively.

\section{DISCUSSION}

Clinical assessment of LV wall motion is mostly based on visual interpretation of dynamic ultrasound images and, more recently, CMR images. While echocardiographic images are usually acquired in dedicated cardiac imaging laboratories and interpreted by cardiologists trained in the evaluation of LV wall motion, CMR imaging is commonly performed in radiology departments and images are frequently reviewed by physicians without dedicated training in cardiology and with variable levels of experience. As a result, the detection of regional wall motion abnormalities from CMR images may be inaccurate and suffer from high inter-observer variability, as indeed confirmed by our results.

To overcome this limitation, we developed and tested a new technique for automated interpretation of $\mathrm{LV}$ wall motion based on the calculation of RFAC and comparisons to segment- and slice-specific threshold values. These threshold values were calculated for each patient from data obtained in a group of normal volunteers, since regional LV wall motion patterns vary from segment to segment (17) and from base to apex, as shown by our results (Fig. 4). The results obtained using these optimized threshold values showed levels of sensitivity, specificity and accuracy around $80 \%$, which are comparable to those seen with other clinically used techniques. Importantly, the automated technique provided more accurate interpretation of regional LV wall motion than the radiologists. We also demonstrated the advantages of the automated interpretation in patients with regional wall motion abnormalities, where accurate interpretation requires even higher levels of expertise, as evidenced by the increased interobserver variability and reduced accuracy achieved by the radiologists in this subgroup of patients.

The improved accuracy of the automated technique was accompanied by another important advantage, ie, the lack of inter-observer variability. Although this technique uses semiautomatically traced endocardial contours, which is likely to introduce a certain level of inter-observer variability, this variability should be only minimal because the high spatial resolution and tissue contrast of CMR images provide excellent endocardial definition. Moreover, semi-automated border detection is available in most commercial software packages and is routinely performed for the evaluation of global LV function. Therefore, our analysis could be easily incorporated into the existing tools and would pose no additional burden in the clinical setting. Most importantly, as the results of this study showed, with virtually no additional user interaction or analysis time beyond the endocardial tracing, this technique can provide accurate objective information on regional LV wall motion. A summary of this information in a bull's eye display allows easy localization of the abnormality and thus may immediately direct the attention to the vascular territory of a specific coronary artery in question. Furthermore, while the bull's eye display only reflects the classification of myocardial segments as normal or abnormal, quantitative information on actual RFAC could also be made available to a clinician in a tabular format, side-by-side with the corresponding normal values, to facilitate the evaluation of the degree of abnormality.

The main limitation of this feasibility study is the relatively small number of patients studied. This approach needs to be tested in the future in a larger group of patients, using a larger sample of the normal population in order to derive more precise reference curves. Another limitation is that the aforementioned variability of the automated interpretation as a result of inter-observer differences in endocardial border tracing was not accessed directly. Although this could be achieved by retracing the images, we chose not to do so for the following reason. Since the variability is likely dependent on the specific border detection technique and since the commercial analysis software does not allow exporting the detected endocardial contours, we could only assess the reproducibility of our border detection technique, and the results of such analysis would not be applicable in a more general sense. It is important to remember however that in this study, border detection was a necessary step toward achieving the goal rather than the goal itself, which was testing the feasibility of automated interpretation of regional wall motion from pre-defined endocardial contours. The reproducibility of automated interpretation from endocardial contours detected by standard analysis packages will have to be determined when testing this combination becomes technically possible.

An additional limitation is that the endocardial border detection technique we used was validated on dynamic CMR images only in a small group of subjects (18). Nevertheless, its ability to correctly identify endocardial borders was favorably tested on contrast-enhanced echocardiographic images (16), where endocardial visualization is affected by intrinsically lower spatial and contrast resolution as well as by acoustic attenuation artifacts. In this regard, SSFP images provide far superior conditions for endocardial identification.

Another limitation is that regional wall motion was classified as normal or abnormal, without differentiating the degree of abnormality. The feasibility and impact of using multiple thresholds in each segment to automatically determine the degree of 
LV wall motion abnormalities requires future investigation. In addition, the fact that the automated interpretation was based on analysis of endocardial displacement alone $(4,5)$, without taking into account systolic myocardial thickening (6-8), could be viewed as a methodological limitation. However, our results showed that even such limited analysis allowed more accurate interpretation than the non-expert readers who used both types of information for their visual interpretation.

In conclusion, automated quantitative analysis of LV regional endocardial motion from CMR images is feasible and provides the basis for accurate detection of regional wall motion abnormalities. The major strength of this approach is that once the endocardial boundaries are defined, the regional analysis and the interpretation of the resultant information are fully automated, immediate, objective and experience-independent. Therefore, this technique could become a clinically valuable addition in the diagnosis of ischemic heart disease.

\section{REFERENCES}

1. Longmore $\mathrm{DB}$, Klipstein $\mathrm{RH}$, Underwood SR, Firmin $\mathrm{DN}$, Hounsfield GN, Watanabe M, Bland C, Fox K, Poole-Wilson PA, Rees RS. Dimensional accuracy of magnetic resonance in studies of the heart. Lancet 1985;1:1360-62.

2. Barkhausen J, Ruehm SG, Goyen M, Buck T, Laub G, Debatin JF. MR evaluation of ventricular function: True fast imaging with steady-state precession versus fast low-angle shot cine MR imaging: Feasibility study. Radiology 2001;219:264-9.

3. Myerson SG, Bellenger NG, Pennell DJ. Assessment of left ventricular mass by cardiovascular magnetic resonance. Hypertension 2002;39:750-5.

4. Underwood SR, Rees RS, Savage PE, Klipstein RH, Firmin DN, Fox KM, Poole-Wilson PA, Longmore DB. Assessment of regional left ventricular function by magnetic resonance. Br Heart J 1986;56:334-40.

5. Pflugfelder PW, Sechtem UP, White RD, Higgins CB. Quantification of regional myocardial function by rapid cine MR imaging. Am J Roentgenol 1988;150:523-9.

6. Holman ER, Vliegen HW, van der Geest RJ, Reiber JH, van Dijkman PR, van der LA, de RA, van der Wall EE. Quantitative analysis of regional left ventricular function after myocardial infarction in the pig assessed with cine magnetic resonance imaging. Magn Reson Med 1995;34:161-9.

7. Peshock RM, Rokey R, Malloy GM, McNamee P, Buja LM, Parkey RW, Willerson JT. Assessment of myocardial systolic wall thickening using nuclear magnetic resonance imaging. J Am Coll Cardiol 1989;14:653-9.
8. Sechtem U, Sommerhoff BA, Markiewicz W, White RD, Cheitlin $M D$, Higgins $C B$. Regional left ventricular wall thickening by magnetic resonance imaging: Evaluation in normal persons and patients with global and regional dysfunction. Am J Cardiol 1987;59:145-511.

9. Picano E, Lattanzi F, Orlandini A, Marini C, L'Abbate A. Stress echocardiography and the human factor: The importance of being expert. J Am Coll Cardiol 1991;17:666-9.

10. Popp RL, Agatston A, Armstrong WF, Nanda NC, Pearlman A, Rakowski, Seward JB, Silverman NH, Smith M, Stewart WJ, Taylor R, Thys D, Davis C. Recommendations for training in performance and interpretation of stress echocardiography. J Am Soc Echocardiogr 1998;11:95-6.

11. Nagel E, Lehmkuhl HB, Bocksch W, Klein C, Vogel U, Frantz E, Ellmer A, Dreysse S, Fleck E. Noninvasive diagnosis of ischemiainduced wall motion abnormalities with the use of high- dose dobutamine stress MRI: Comparison with dobutamine stress echocardiography. Circulation 1999;99:763-70.

12. Hundley WG, Hamilton CA, Thomas MS, Herrington DM, Salido TB, Kitzman DW, Little WC, Link KM. Utility of fast cine magnetic resonance imaging and display for the detection of myocardial ischemia in patients not well suited for second harmonic stress echocardiography. Circulation 1999;100:1697-702.

13. Caiani EG, Toledo E, MacEneaney P, Collins KA, Lang RM, Mor-Avi $V$. The role of still-frame parametric imaging in magnetic resonance assessment of left ventricular wall motion by non-cardiologists. $J$ Cardiovasc Magn Reson 2004;6:619-25.

14. Schiller NB, Shah PM, Crawford M, DeMaria A, Devereux R, Feigenbaum H, Gutgessel H, Reichek N, Sahn D, Schnittger I, Silverman NH, Tajik AJ. Recommendations for quantitation of the left ventricle by two-dimensional echocardiography. J Am Soc Echocardiogr 1989;2:358- 67.

15. Guidelines for credentialing in cardiovascular magnetic resonance (CMR). Society for Cardiovascular Magnetic Resonance (SCMR) Clinical Practice Committee. J Cardiovasc Magn Reson 2000;2:233-4.

16. Caiani EG, Lang RM, Caslini S, Collins KA, Korcarz CE, MorAvi V. Quantification of regional myocardial perfusion using semiautomated translation-free analysis of contrast-enhanced power modulation images. J Am Soc Echocardiogr 2003;16: 116-23.

17. Mor-Avi V, Spencer K, Gorcsan J, DeMaria A, Kimball T, Monaghan M, Perez J, Sun JP, Weinert L, Bednarz J, Collins K, Edelman K, Kwan OL, Glascock B, Hancock J, Baumann C, Thomas J, Lang $R$. Normal values of regional left ventricular endocardial motion: Multicenter color kinesis study. Am J Physiol Heart Circ Physiol 2000;279:H2464-76.

18. Caiani EG, Toledo E, MacEneaney P, Collins KA, Lang RM, Mor-Avi V. Objective assessment of left ventricular wall motion from cardiac magnetic resonance images. IEEE Transac Computers Cardiol; 2004 p. 153-6. 